\title{
Breaking Discrete Symmetries in Broken Gauge Theories
}

\author{
Thomas Appelquist, Yang Bai, and Maurizio Piai \\ Physics Department, Sloane Laboratory \\ Yale University \\ New Haven, CT 06520
}

\begin{abstract}
We study the spontaneous breaking of discrete symmetries in theories with broken gauge symmetry. The intended application is to CP breaking in theories with gauged flavor symmetries, but the analysis described here is preliminary. We dispense with matter fields and take the gauge theory to be weakly coupled and broken spontaneously by unspecified, short-distance forces. We develop an effective-field-theory description of the resultant low energy theory, and ask whether this theory by itself can describe the subsequent breaking of discrete symmetries. We conclude that this can happen depending on the parameters of the effective theory, and that the intrinsic violation is naturally of order unity.
\end{abstract}

\section{INTRODUCTION}

The array of quark and lepton masses and mixing angles, including CP violation, remains a mystery. Its resolution may involve a spontaneously broken, non-abelian flavor symmetry, a feature common to many approaches. For example, an $S U(3)$ family symmetry [1, 2] can be appended onto the standard model or its supersymmetric extension. The flavor symmetry could be even larger than $S U(3)$, incorporating additional, heavy generations, a typical feature of extended technicolor models. If the flavor symmetry is gauged, the interactions with the (broken) gauge sector may be responsible for the observed features of the quark and lepton mass matrices.

$\mathrm{CP}$-violation is one of these features. Of all the terms in the standard-model (SM) (dimension-4) Lagrangian which could violate $\mathrm{CP}$, those that appear in connection with flavor-changing currents contain an $O(1) \mathrm{CP}$ violating phase (the CKM phase $\delta$ ). The phase associated with flavor-diagonal processes is extremely small ( $\bar{\theta}$ in the strong $\mathrm{CP}$ problem). This suggests that the measured CP violation could be associated with a flavor gauge group, and that spontaneous breaking of the latter may lead to the breaking of discrete symmetries such as CP, and ultimately control CP-violation in flavor changing processes [3, 4, [5].

In this paper, we study more generally the spontaneous breaking of discrete symmetries in spontaneously broken gauge theories. Our main focus is on an $S U(3)$ gauge theory, where the $S U(3)$ may be thought of as flavor group. We consider only the gauge sector, dispensing with the standard-model fields. Because the vacuum structure of such a theory is closely related to the $8 \times 8$ (real symmetric) gauge-boson mass matrix, we find it natural to embed the $S U(3)$ gauge theory in a larger model with an $S O(8)$ global symmetry. We then ask whether the resultant effective low energy theory can, by itself, describe the spontaneous breaking of the discrete symmetry.

To set the stage, we first examine a simpler example in which an $S O(2)$ gauge theory is embedded in a model with a global $S O(3)$ symmetry. Although this embedding is not as compelling as $S U(3)$ in $S O(8)$, the model shares many features with the $S O(8)$ model, including discrete symmetries, and its vacuum structure can be more easily and thoroughly studied. After describing this study, we examine the $S U(3)$ theory. In each case, since we are interested in a limited range of energies, we neglect $\mathrm{RG}$ running of the gauge couplings.

In Section II, we describe the $S O(2)$ gauge theory embedded in $S O(3)$. With the $S O(3)$ broken spontaneously and with the gauge coupling sufficiently weak, the effective low-energy theory involves a single massive gauge field along with two massive scalars. They are psuedo Nambu-Goldstone bosons (PNGB's). We identify the discrete symmetry of the model, and analyze the symmetry structure of the vacuum by studying a scalar potential in terms of the PNGB fields. A vacuum phase diagram emerges with the resultant discrete symmetry depending on the relative strength of different terms. We note that the phases can be physically distinguished through certain selection rules.

In Section III, we turn to the $S U(3)$ gauge theory. We describe its $S O(8)$ embedding and the discrete symmetry of the model. A complete spontaneous breaking of the $S O(8)$ symmetry leads to 8 Goldstone fields and 20 additional scalar fields which are massive due to the explicit breaking of $S O(8)$ to $S U(3)$ by the gauge interaction. With $g$ sufficiently weak, the 20 scalars are PNGB's, with masses small relative to the breaking scale. The resultant low energy effective theory contains the PNGB's and the 8 massive gauge fields. We describe the operators of this theory, and study the vacuum structure in terms of the PNGB fields to determine under what conditions the discrete symmetry is broken spontaneously. With 20 PNGB fields, the analysis is more intricate than in the $S O(2)$ case. We conclude again, however, that the spontaneous breaking of the discrete symmetries can be driven by the low energy effective theory depending on the relative size of certain low-energy parameters.

We summarize in Section IV, and discuss open questions. 


\section{ANALYSIS OF AN $S O(2)$ GAUGE THEORY, EMBEDDED IN $S O(3)$}

The three generators for $S O(3)$ may be taken to be

$$
\left(T_{i}\right)_{j k}=-i \epsilon_{i j k} .
$$

We choose $T_{3}$ as the generator of the gauged $S O(2)$. We employ a set of scalar fields transforming as the symmetric traceless rank- 2 tensor, here the 5 representation of $S O(3)$, denoted $\Sigma_{a b}, a, b=1,2,3$. We assume that some underlying dynamics at a scale $\Lambda$ spontaneously breaks the global $S O(3)$ completely, producing a VEV for $\Sigma_{a b}$.

To describe physics below the scale $\Lambda$, we freeze out two of the five degrees of freedom in $\Sigma$, leaving the three Goldstone degrees of freedom. This can be done via the two nonlinear constraints

$$
\operatorname{Tr} \Sigma^{2}=f^{2} / 2
$$

and

$$
\operatorname{Tr} \Sigma^{3}=A f^{3}
$$

where $\Lambda=O(4 \pi f)$ and where $A$ is a dimensionless parameter with unit magnitude. The first constraint is invariant under a larger, $S O(5)$, symmetry among the components of $\Sigma$, while the latter is $S O(3)$ symmetric.

If the $S O(2)$ gauge coupling were not present, the VEV could be diagonalized by a general $S O(3)$ transformation to take the matrix form

$$
F=\operatorname{diag}(a, b, c),
$$

where $a+b+c=0$. We then have $a^{2}+b^{2}+c^{2} \equiv f^{2} / 2$. For general values $a \neq b \neq c$, the $S O(3)$ symmetry is completely broken.

In the presence of the $S O(2)$ gauge coupling, the terms in the effective low-energy Lagrangian quadratic in the covariant derivative are

$$
\mathrm{E}=-\frac{1}{4} F_{\mu \nu} F^{\mu \nu}+\frac{1}{2} \operatorname{Tr}\left(D_{\mu} \Sigma\right)^{2}+\frac{d}{2 f^{2}} \operatorname{Tr}\left(D_{\mu} \Sigma^{2}\right)^{2},
$$

where $D_{\mu} \Sigma^{n}=\partial_{\mu} \Sigma^{n}+i g A_{\mu}\left[T_{3}, \Sigma^{n}\right]$ and $d$ is a dimensionless constant. That the most general two-derivative lagrangian can be written in terms of just these operators can be shown by making use of the nonlinear constraints (2) and (3). These allow the quantity $\Sigma^{n}$ for $n \geq 3$ to be written as a linear combination of $\Sigma, \Sigma^{2}$ and the identity matrix. This together with a simple point transformation leads to the above Lagrangian. Thus, the two-derivative Lagrangian is characterized by the gauge coupling along with three additional parameters, $f, A$, and $d$.

To these terms must be added higher-derivative operators, as well as operators with no derivatives describing the mass and interactions of the PNGB's. To study this model within the framework of effective field theory, we take the gauge coupling $g$ to be small $\left(g^{2} / 16 \pi^{2} \ll 1\right)$, preserving an approximate $S O(3)$ symmetry.
Only one of the three Goldstone fields in $\Sigma$ is massless; for weak gauge coupling the other two are PNGB's with masses of order $g f \ll \Lambda$. To express the VEV of $\Sigma$, only an $S O(2)$ transformation generated by $T_{3}$ is now available to rotate it. This freedom may be used to write the VEV in the general form

$$
\langle\Sigma\rangle \equiv \Sigma_{0}=e^{i \hat{\phi}_{1} T_{1}+i \hat{\phi}_{2} T_{2}} F e^{-i \hat{\phi}_{1} T_{1}-i \hat{\phi}_{2} T_{2}},
$$

where $\hat{\phi}_{1}$ and $\hat{\phi}_{2}$ represent (space-time independent) VEV's of the PNGB fields. The compact domain for these two parameters can be determined to be

$$
0 \leq \hat{\phi}_{1}^{2}+\hat{\phi}_{2}^{2}<\pi^{2} .
$$

It can be seen that all points along the boundary $\hat{\phi}_{1}^{2}+$ $\hat{\phi}_{2}^{2}=\pi^{2}$ are all equivalent to the origin up to an $S O(2)$ gauge transformation generated by $T_{3}$.

A convenient form for $\Sigma$ is

$$
\Sigma=e^{i \phi_{1} T_{1}+i \phi_{2} T_{2}+i \phi_{3} T_{3}} \Sigma_{0} e^{-i \phi_{1} T_{1}-i \phi_{2} T_{2}-i \phi_{3} T_{3}},
$$

where the $\phi_{a}$ are the quantum fields, defined to have vanishing VEV's. Using this expression and keeping only terms in $\mathrm{E}$ quadratic in the quantum fields, we have

$$
\begin{aligned}
\mathrm{E}_{2}= & -\frac{1}{4} F_{\mu \nu} F^{\mu \nu}+\frac{1}{2} \partial \phi_{a} K_{a b} \partial \phi_{b} \\
& +g W_{a} \partial_{\mu} \phi_{a} A^{\mu}+\frac{1}{2} A_{\mu} M^{2} A^{\mu},
\end{aligned}
$$

with

$$
M^{2}(\hat{\phi})=-g^{2} \operatorname{Tr}\left[T_{3}, \Sigma_{0}\right]\left[T_{3}, \Sigma_{0}\right]+\ldots,
$$

and with similar expressions for $K(\hat{\phi})_{a b}$ and $W_{a}$. The dots represent the contribution of the third term in the Lagrangian (5). From the explicit expression (6) for $\Sigma_{0}$, it can be seen that this term gives the same dependence on the VEV's of PNGB fields as the term shown, along with an additive constant.

One can introduce canonically normalized scalar fields $\varphi_{a}$ with $a=1,2,3$, by diagonalizing the scalar kinetic term with a non-orthogonal transformation of the quantum fields $\phi_{a}=Q_{a b} \varphi_{b}$ such that $Q^{T} K Q=1$, where $Q$ is a $3 \times 3$ constant matrix with inverse-mass dimension. Identifying $\varphi_{3}$ with the component that mixes with the gauge boson, we have

$$
\begin{aligned}
\mathrm{E}_{2}= & -\frac{1}{4} F_{\mu \nu} F^{\mu \nu}+\frac{1}{2} \partial \varphi_{a} \partial \varphi_{a} \\
& +g W_{a} Q_{a 3} \partial_{\mu} \varphi_{3} A^{\mu}+\frac{1}{2} A_{\mu} M^{2} A^{\mu}
\end{aligned}
$$

Thus $\varphi_{1}$ and $\varphi_{2}$ are the two PNGB's.

\section{A. Discrete Symmetries}

If the $S O(2)$ gauge coupling were not present, the symmetry of the nonlinear Lagrangian (5) would be $S O(3)$ 
along with a discrete $Z_{2}$ symmetry which can be taken to be $\Sigma \rightarrow C_{1} \Sigma C_{1}^{-1}$, where

$$
C_{1}=\operatorname{diag}(-1,+1,+1) \text {. }
$$

The $S O(3)$-breaking vacuum, described by the diagonal $F$ matrix (4), would have two independent discrete symmetries which can be taken to be $C_{1}$ and

$$
C_{2}=\operatorname{diag}(+1,-1,+1) .
$$

Other discrete transformations can be formed by composing these two along with $C_{-}=\operatorname{diag}(-1,-1,-1)$, which is the identity transformation on $\Sigma$. Thus the discrete $Z_{2}$ symmetry of the Lagrangian would remain unbroken in the vacuum.

In the presence of the $S O(2)$ gauge coupling, the Lagrangian is invariant under the discrete transformation

$$
\begin{aligned}
\Sigma & \rightarrow C_{1} \Sigma C_{1}^{-1} \\
A_{\mu} & \rightarrow-A_{\mu} .
\end{aligned}
$$

Other discrete symmetries are equivalent to this one together with a global $S O(2)$ transformation.

The possible discrete symmetries of the vacuum state, described by $\Sigma_{0}$ (6), depend on the values of $\hat{\phi}_{1}$ and $\hat{\phi}_{2}$. If both vanish, then $\Sigma_{0}=F$, and the vacuum has two discrete symmetries which, as in the absence of the gauge interaction, can be taken to be $C_{1}$ and $C_{2}$. The same is true for other values of $\hat{\phi}_{1}$ and $\hat{\phi}_{2}$ that amount to only a permutation of the entries of $F$. For a generic value of $\hat{\phi}_{1}$ but with $\hat{\phi}_{2}=0, \Sigma_{0}$ is invariant under the $C_{1}$ transformation. Similarly, with $\hat{\phi}_{1}=0$ but generic $\hat{\phi}_{2}$, the vacuum symmetry is $C_{2}$. For generic values of both $\hat{\phi}_{1}$ and $\hat{\phi}_{2}, C_{1}$ and $C_{2}$ are broken, and the vacuum has no symmetry, continuous or discrete.

\section{B. Additional Operators}

We append to the effective Lagrangian (5) additional local operators consistent with its symmetries. Since we are interested in the contribution of these operators to the classical potential, and since the VEV of $A_{\mu}$ must vanish, we restrict attention to operators involving only $\Sigma$ (8), with no covariant derivatives. These will naturally be generated by unknown high energy physics beyond the cutoff $\Lambda$, and also in the loop expansion of the Lagrangian (5). They require the explicit breaking of the global $S O(3)$, and with the assumption that the only such breaking, whether above or below $\Lambda$, arises from the gauge interaction, they must enter with powers of $g$.

We begin with operators including two factors of $T_{3}$, which are generated at order $g^{2}$ from the Lagrangian (5). By making use of the nonlinear constraints (2) and (3), they can all be written in terms of

$$
O_{2}=\operatorname{Tr}\left[T_{3}, \Sigma\right]\left[T_{3}, \Sigma\right]
$$

together with $\operatorname{Tr}\left[T_{3}, \Sigma\right]\left[T_{3}, \Sigma^{2}\right]$ and $\operatorname{Tr}\left[T_{3}, \Sigma^{2}\right]\left[T_{3}, \Sigma^{2}\right]$. All are $S O(2) \times C_{1}$ invariant. From the explicit form of $\Sigma(8)$, it can be seen that the second two operators give the same dependence on the PNGB fields as $\mathrm{O}_{2}$ up to an additive constant. We don't consider them further. Since $\mathrm{O}_{2}$ enters at order $g^{2}$ in the low energy effective theory (5), we take this to be the case in general. In the low energy theory, it arises in Landau gauge from a single gauge-boson loop, with a coefficient quadratically sensitive to the cutoff $\Lambda$.

\section{Vacuum Alignment in the Simplest Case}

Replacing $\Sigma$ by its vacuum value $\Sigma_{0}$, the operator $O_{2}$ gives the potential

$$
V_{2}=-C g^{2}\left(\Lambda^{2} / 16 \pi^{2}\right) \operatorname{Tr}\left[T_{3}, \Sigma_{0}\right]\left[T_{3}, \Sigma_{0}\right],
$$

where $\Sigma_{0}$ is given by Eq. (6), and $C$ is taken to be of unit magnitude and positive, as suggested by the low energy calculation. In terms of these parameters and the elements of $F$ (4), the explicit form of the potential is

$$
\begin{aligned}
V_{2}= & C g^{2}\left(\Lambda^{2} / 8 \pi^{2}\right)\left((a-b)^{2}+\right. \\
& \left.3 \frac{a(a+2 b) \hat{\phi}_{1}^{2}+b(b+2 a) \hat{\phi}_{2}^{2}}{\hat{\phi}_{1}^{2}+\hat{\phi}_{2}^{2}} \sin ^{2} \sqrt{\hat{\phi}_{1}^{2}+\hat{\phi}_{2}^{2}}\right)
\end{aligned}
$$

Recall that the domain is given by Eq. (7). In addition to $S O(2) \times C_{1}$, this potential is invariant under reflection about the circle $\hat{\phi}_{1}^{2}+\hat{\phi}_{2}^{2}=\pi^{2} / 4$.

Minimization of $V_{2}$ provides a simple example of vacuum alignment. Any ordering of the parameters $a, b$, and $c(=-a-b)$ in $V_{2}$ may be adopted at the outset. It is convenient, however, to order them such that $(a-b)^{2}$ is the smallest squared difference, that is, $(a-b)^{2} \leq(a-c)^{2},(b-c)^{2}$. This corresponds to taking $a$ and $b$ to be the entries with the same sign. It can then be seen that $V_{2}$ is minimized by the choice $\hat{\phi}_{1}=\hat{\phi}_{2}=0$. Since the gauge boson mass $M^{2}(\hat{\phi})$ is proportional to $V_{2}$ up to an additive constant, minimizing $V_{2}$ makes the gauge boson mass as small as possible. Other ordering choices would lead to the same result since the $\hat{\phi}_{i}$ would then take on different vacuum values, either 0 and $\pm \pi / 2$ or $\pm \pi / 2$ and 0 , that simply permute the $F$ entries $a, b$, and $c$ in $\Sigma_{0}$. All these values for $\hat{\phi}_{1}$ and $\hat{\phi}_{2}$ are equivalent, and leave the vacuum invariant under the two discrete symmetries $C_{1}$ and $C_{2}$.

We note that if any two of the $F$ entries $a, b$, and $c$ are degenerate, the spontaneous breaking of the $S O(3)$ leaves a residual $S O(2)$ symmetry group, which aligns with the gauged $S O(2)$ subgroup, yielding a massless gauge boson. Again, the vacuum is invariant under the discrete symmetries $C_{1}$ and $C_{2}$. 


\section{Higher-Order Operators}

To make possible a pattern of spontaneous discrete symmetry breaking in this model, additional potential terms must be included along with $V_{2}$ (16). These come from operators with more than two $T_{3}$ factors, hence arising at higher orders in $g^{2}$. Higher powers of the operator $\mathrm{O}_{2}$ are of no interest, since they enter with higher powers of $g^{2}$ and are therefore always suppressed for small gauge coupling. There are other operators entering at order $g^{4}$ and above, however, which are typically small compared to the $\mathrm{O}\left(g^{2}\right)$ operators, but have a different dependence on the parameters of $F$. For certain choices of these parameters, the $\mathrm{O}\left(g^{2}\right)$ operators are suppressed, making these new operators at least comparable. Among them, one is bilinear in $\Sigma$, and can be taken to be

$$
O_{4}=\operatorname{Tr}\left[T_{3},\left[T_{3}, \Sigma\right]\right]\left[T_{3},\left[T_{3}, \Sigma\right]\right]
$$

It is directly related to the four derivative operator $\operatorname{Tr}\left(D^{2} \Sigma\right)^{2}$ which must be added to the Lagrangian (5); by using this four-derivative term and integrating out the gauge boson fields, $\mathrm{O}_{4}$ is generated.

Replacing $\Sigma$ by $\Sigma_{0}$, we denote the corresponding classical potential by $V_{4}$, and use it as a representative of potential terms with four factors of $T_{3}$. We then study the effective potential

$$
\begin{aligned}
V= & V_{2}+V_{4}, \\
= & \frac{-C g^{2} \Lambda^{2}}{16 \pi^{2}}\left\{\operatorname{Tr}\left[T_{3}, \Sigma_{0}\right]\left[T_{3}, \Sigma_{0}\right]\right. \\
& \left.+B \operatorname{Tr}\left[T_{3},\left[T_{3}, \Sigma_{0}\right]\right]\left[T_{3},\left[T_{3}, \Sigma_{0}\right]\right]\right\},
\end{aligned}
$$

where $B=O\left(g^{2} / 16 \pi^{2}\right)$.

To analyze this potential, we introduce the notation

$$
F=\frac{f}{\sqrt{3}} \operatorname{diag}\left(\cos \left(\alpha-\frac{\pi}{3}\right), \cos \left(\alpha+\frac{\pi}{3}\right),-\cos (\alpha)\right)(20)
$$

The ordering convention $(a-b)^{2} \leq(b-c)^{2},(a-c)^{2}$ then reduces to

$$
-\frac{\pi}{6} \leq \alpha \leq \frac{\pi}{6}
$$

(The interval $[5 \pi / 6,7 \pi / 6]$ is equivalent, up to a change of sign of $f$ which does not affect the potential.)

The symmetries of $V=V_{2}+V_{4}$ are the same as those of $V_{2}: S O(2), C_{1}$, and a reflection symmetry about the circle $\hat{\phi}_{1}^{2}+\hat{\phi}_{2}^{2}=\pi^{2} / 4$. These symmetries allow us to restrict our study to the region

$$
\left\{\begin{array}{c}
\hat{\phi}_{i} \geq 0 \\
\sqrt{\hat{\phi}_{1}^{2}+\hat{\phi}_{2}^{2}} \leq \pi / 2
\end{array}\right.
$$

Analysis of $V$ as a function of $B$ and $\alpha$ reveals a variety of phases with respect to the discrete symmetries, in the range $B \leq 0$. This is the range suggested by an estimate from low-energy physics cutoff at $\Lambda$. The phases are described by Fig. 1. We do not write the explicit form of the potential, but simply note that for $C>0$, it has a global minimum at either the origin or along the $\hat{\phi}_{1}=0$ or $\hat{\phi}_{2}=0$ axes, depending on the location in the $\alpha, B$ plane.

On the right side of Fig. $1(0 \leq \alpha \leq \pi / 6)$, the vacuum solution is $\hat{\phi}_{1}=0$, but $\hat{\phi}_{2}$ depends on the parameters $B$, $\alpha$. To describe the shape of the boundary curve there, we note that when $\hat{\phi}_{1}=0$, the potential $V$ takes the form

$$
\begin{aligned}
V= & \frac{C g^{2} f^{2} \Lambda^{2}}{32 \pi^{2}}\left\{(4-16 B) \sin ^{2} \alpha\right. \\
+ & {[3 \cos 2 \alpha-\sqrt{3} \sin 2 \alpha} \\
& +B(-9 \cos 2 \alpha+7 \sqrt{3} \sin 2 \alpha+6)] \sin ^{2} \hat{\phi}_{2} \\
- & \left.3 B(\cos 2 \alpha+\sqrt{3} \sin 2 \alpha+2) \sin ^{4} \hat{\phi}_{2}\right\},
\end{aligned}
$$

with an analogous expression for the left side.

For a potential of the general form $k_{0}+k_{2} \sin ^{2} \hat{\phi}_{2}+$ $k_{4} \sin ^{4} \hat{\phi}_{2}$, a simple argument leads to the conclusion that for $k_{2}<0$ and $k_{4}>-1 / 2 k_{2}$, one has a $C_{1}$-violating minimum at $\hat{\phi}_{2}= \pm(1 / 2) \arccos \left(\left(k_{2}+k_{4}\right) / k_{4}\right)$. This procedure, with a similar one for the left side of Fig. 1, leads directly to a phase diagram in the $\alpha, B$ plane, with the boundary given by the expression

$$
B^{*}=\frac{3 \cos 2 \alpha \pm \sqrt{3} \sin 2 \alpha}{9 \cos 2 \alpha \pm 7 \sqrt{3} \sin 2 \alpha-6} .
$$

The boundary is a line of second-order phase transitions.

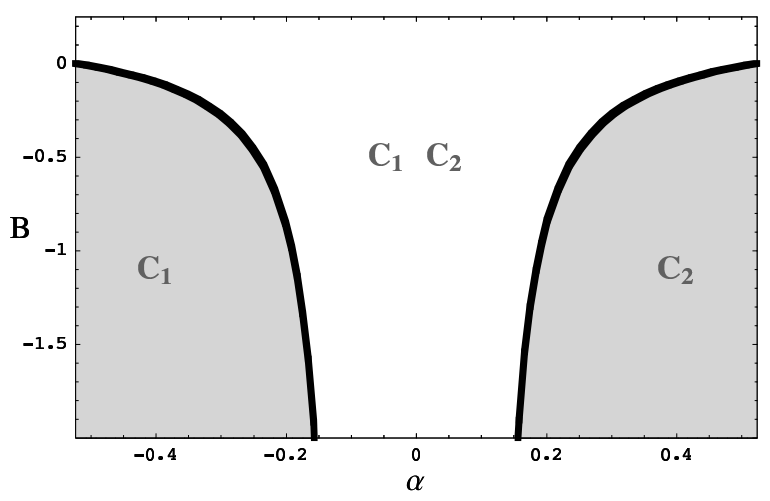

FIG. 1: Discrete-symmetry phases in the $\alpha, B$ plane. In the light area, the minimum of the potential is at the origin. In the dark area the minimum lies along one of the $\hat{\phi}_{i}=0$ axes. Each region is labelled by its discrete symmetries. The boundary, given by Eq. (24), is a line of second-order phase transitions

The discrete symmetry of the vacuum when $\hat{\phi}_{1}=\hat{\phi}_{2}=$ 0 (the light region of Fig. 11) is $C_{1} \times C_{2}$. In the right-hand dark region, the vacuum corresponds to the axis $\hat{\phi}_{1}=0$, and the discrete symmetry is only $C_{2}$. As the right-right dark boundary line is approached, the field $\hat{\phi}_{2}$, which is odd under $C_{1}$, becomes massless. By integrating out the 
gauge boson and $\hat{\phi}_{1}$ fields, the transition can be described by a Landau-Ginsberg potential in $\hat{\phi}_{2}$. The transition along the left boundary is the same, with $\hat{\phi}_{2} \rightarrow \hat{\phi}_{1}$ and $C_{2} \rightarrow C_{1}$.

The physical distinction between the phases of Fig. 11 can be characterized in terms of the quantum fields of Eq. (11). With the operators $O_{2}$ and $O_{4}$ added to the Lagrangian (5), and after properly normalizing the two physical scalar fields $\varphi_{i}$ as in Eq. (11):

- Both $\varphi_{i}$ fields have positive-definite masses, one of which vanishes along the line $B=B^{*}$ of secondorder phase transitions.

- In the light region of Fig. 11 where the vacuum is at $\hat{\phi}_{1}=\hat{\phi}_{2}=0$, the Lagrangian has terms containing only even powers of both quantum $\varphi_{i}$ fields. The model realizes two discrete symmetries $\varphi_{i} \rightarrow \pm \varphi_{i}$, and there is a double selection rule. In any scattering process, the number of $\varphi_{1}$ particles and $\varphi_{2}$ particles is conserved mod 2 .

- In either dark region of Fig. 1 the Lagrangian is invariant under only one discrete symmetry $\left(C_{1}\right.$ or $C_{2}$ ), and terms that are odd in the other are present. Only one selection rule is present. One type of particle is conserved mod 2, while the other is not conserved.

\section{E. Discussion}

Because $B=O\left(g^{2} / 16 \pi^{2}\right) \ll 1$, the parameter range of special interest here is $\alpha \approx+\pi / 6$, corresponding to the hierarchy $|b| \ll|a|,|c|$. (The choice $\alpha \approx-\pi / 6$ (the hierarchy $|a| \ll|b|,|c|)$ is equivalent.) With only the operator $\mathrm{O}_{2}$, the effective potential is given by $V_{2}$, and one can see from Eq. (17) that in this range the vacuum always corresponds to $\hat{\phi}_{1}=0$. The potential becomes flat in $\hat{\phi}_{2}$ in the limit. Thus other operators are expected to be important in this special case. We have analyzed $\mathrm{O}_{4}$ as an example.

For $\alpha$ close to $+\pi / 6$, the potential $V=V_{2}+V_{4}$ produces a second-order phase transition for small $B$, from the phase with vacuum symmetry $C_{1} \times C_{2}$ to one with only $C_{2}$. With $C_{1}$ spontaneously broken, there is then no (relative) small parameter in $V$, and the value of $\hat{\phi}_{2}$ is generically of order unity; the intrinsic breaking is large. On the other hand, the manifestation of this effect through selection-rule violating terms is suppressed by a small "mixing angle" - specifically $|b / a| \approx|b / c|$.

Thus, with the small $g$ required for a consistent effective field theory, there is a range of parameter space which allows a set of different discrete-symmetry phases. Furthermore, the model is predictive, meaning that the effective low energy theory can be described by a small number of parameters $(f$ and $\alpha$, the gauge coupling $g$, and the potential parameters). The measurement of the masses of the three physical particles and a set of $C_{i}$-even couplings can determine all of these parameters. One can then predict which discrete-symmetry phase is realized.

\section{AN $S U(3)$ GAUGE THEORY, EMBEDDED IN $S O(8)$}

The analysis of discrete symmetries in this more realistic gauge theory is more intricate than in the $S O(2)$ example. While the discussion here is less complete, the features that have emerged so far are similar in many respects to $S O(2)$.

We take the $S O(8)$ model to be described by a set of scalar fields (composite or fundamental) transforming according to the 35 representation - a symmetric, traceless, rank-2 tensor denoted by $S_{a b}, a, b=1, \cdots, 8$. We assume the global symmetry to be completely spontaneously broken by some unspecified underlying dynamics producing a vacuum expectation value (VEV) for $S_{a b}$. An effective low-energy description of this theory, valid up to the $S O(8)$ symmetry breaking scale $\Lambda=O(4 \pi f)$ and containing only the 28 Goldstone fields from the 35 , can be constructed by freezing out the 7 massive components of $S$. This corresponds to imposing a set of 7 constraints on $S$, for example $\operatorname{Tr} S^{2}=f^{2} / 2$.

We gauge an $S U(3)$ subgroup of $S O(8)$. A simple way to embed $S U(3)$ in $S O(8)$ is to identify the 8 generators $\left(t_{a}\right)_{b c}=-i f_{a b c}$ of $S U(3)$ in the adjoint representation, and complete this to an orthonormal basis for $S O(8)$, by adding twenty $8 \times 8$ matrices $s_{b}, b=9, \cdots, 28$. This corresponds to choosing the $S U(3)$ generators to form a maximal subalgebra of $S O(8)$. We denote the complete generator basis as $x_{c}=\left(t_{a}, s_{b}\right)$. In terms of $S U(3)$, the 28 decomposes into $8+10+\overline{10}$. Since the $S O(8)$ is completely spontaneously broken, so too is the $S U(3)$, and the 8 in this decomposition is the set of Goldstone bosons which are eaten to produce the longitudinal component of the gauge bosons.

We write

$$
\mathrm{E}=-\frac{1}{2} \operatorname{Tr} F_{\mu \nu} F^{\mu \nu}+\frac{1}{2} \operatorname{Tr}\left(D_{\mu} S\right)^{2}+\cdots,
$$

where

$$
D^{\mu} S=\partial^{\mu} S+i g\left[A^{\mu}, S\right] .
$$

The gauge field $A_{b c}^{\mu}=A_{a}^{\mu}\left(t_{a}\right)_{b c}$ is in the $S U(3)$ adjoint representation. The dots in Eq. (25) represent additional operators involving $S$ and the gauge fields, that preserve the gauged $S U(3)$ and other symmetries of $\mathrm{E}$. There are terms quadratic in the covariant derivative $D^{\mu}$, terms involving higher derivatives, and the zero-derivative terms of special interest here.

We parametrize the VEV $\langle S\rangle$ by introducing the notation $\hat{\phi}=\hat{\phi}_{c} x_{c}$, where the $\hat{\phi}_{c}$ are 28 real parameters (the VEV's of the Goldstone fields), and writing

$$
\langle S\rangle \equiv S_{0}=e^{i \hat{\phi}} F e^{-i \hat{\phi}} .
$$


Here, $F=\operatorname{diag}\left(f_{a}\right)$ where the $f_{a}$ are eight real massparameters with $\sum f_{a}=0$.

The quantum field $S$ can then be expressed in the nonlinear form

$$
S=e^{i \phi} S_{0} e^{-i \phi},
$$

with $\phi=\phi_{c} x_{c}$, where the $28 \phi_{c}$ are the Goldstone fields, defined to have vanishing vacuum expectation values. Since $S U(3)$ is a maximal subgroup of $S O(8)$, there are no global continuous residual symmetries, and hence all 20 components of $\phi$ in the $S O(8) / S U(3)$ coset acquire a radiatively generated mass and a non-trivial potential, due to the gauge interactions. The gauge coupling is taken to be weak enough $\left(g^{2} / 16 \pi^{2} \ll 1\right)$ so that the scalar masses and the gauge boson masses, both of order $g f$ are small compared to the cutoff $\Lambda$. The scalars are PNGB's.

The $S O(8)$ embedding is natural in the sense that it describes, for general values of the $28 \hat{\phi}$ fields, the most general ( $S U(3)$-breaking) mass matrix for the $A$ fields, a real, symmetric, $8 \times 8$ matrix. The corresponding term in $\mathrm{E}$, bilinear in $A$, is

$$
\mathrm{E}_{m}=\frac{1}{2} A_{a}^{\mu} \mathcal{M}^{2}(\hat{\phi})_{a c} A_{\mu c}
$$

where

$$
\mathcal{M}^{2}(\hat{\phi})_{a c}=-g^{2} \operatorname{Tr}\left\{\left[t_{a}, S_{0}\right]\left[t_{c}, S_{0}\right]\right\}+\ldots \ldots,
$$

where the dots represent the contribution of the additional two-derivative operators in the Lagrangian (25).

The dependence of $\mathcal{M}^{2}(\hat{\phi})$ on the fields $\hat{\phi}_{a}$ with $a=$ $1 \ldots 8$ may be removed by an $S U(3)$ transformation, leaving it depending on only the seven independent parameters in $F$ and the 20 PNGB's in the $S O(8) / S U(3)$ coset.

\section{A. Discrete Symmetries}

In addition to the gauged $S U(3)$, the Lagrangian is invariant under the action of a discrete symmetry $C$, which can be identified by noting that $S U(3)$ admits one maximal subgroup with a symmetric coset. It is an $S O(3)$ associated with the generators $t_{2}, t_{5}$ and $t_{7}$. Thus, within the $S U(3)$, the action of $C$ is uniquely defined (up to an $S U(3)$ transformation) as:

$$
\begin{aligned}
A_{a}^{\mu} & \rightarrow C_{a b} A_{b}^{\mu}, \\
C & =\operatorname{diag}\{-1,1,-1,-1,1,-1,1,-1\} .
\end{aligned}
$$

This is the canonical definition of charge-conjugation. In terms of the matrix field $A_{a b}^{\mu}=A_{c}^{\mu}\left(t_{c}\right)_{a b}$, the transformation Eq. (31) becomes

$$
A^{\mu} \rightarrow C A^{\mu} C^{-1} \text {. }
$$

For the full $S O(8)$ theory, one can deduce from Eq. (31) the transformation properties under $C$ of all the representations. In particular, the rank-two symmetric, traceless tensor field $S$ transforms as

$$
S \rightarrow C S C^{-1} .
$$

This is an outer-automorphism of $S O(8)$, leaving the Lagrangian (25) invariant. As with $A, S$ is not an irreducible representation of $C$, but decomposes into a $C$ even and $C$-odd part. Among the $28 \phi$ fields in $S$, the eight Goldstone fields transform just as Eq. (31), while of the remaining 20, 10 are $C$-odd and 10 are $C$-even.

\section{B. Additional Operators}

Among the $S U(3) \times C$-invariant operators to be added to the Lagrangian (25), are those that lead to vacuumdetermining potential terms. Since the VEV of $A_{\mu}$ must vanish, these involve only the field $S$ (28). In each of these operators and the associated potential terms, the $S U$ (3) invariance may be used to eliminate dependence on the 8 Goldstone fields. The potential terms then depend on only the 20 PNGB fields $\hat{\phi}_{9} \ldots . . \hat{\phi}_{28}$ in the coset $S O(8) / S U(3)$. The full potential describes a landscape in this 20-dimensional space.

We begin at first order in $g^{2}$, and therefore with two factors of $t_{a}$. One such operator is bilinear in $S$, and can be taken to be

$$
\emptyset_{2} \equiv \operatorname{Tr}\left[t_{a}, S\right]\left[t_{a}, S\right]
$$

It is generated at order $g^{2}$ with quadratic dependence on the cutoff $\Lambda$. (That this is the only potential-generating operator bilinear in $S$ can be seen by noting that $S$, which transforms as a 35 under $S O(8)$, decomposes into $8+27$ under its maximal $S U(3)$ subgroup. There are therefore two independent bilinears, corresponding to $8 \times 8 \rightarrow 1$ and $27 \times 27 \rightarrow 1$. But one is the $S O(8)$ invariant $\operatorname{Tr} S^{2}=f_{a} f_{a} \equiv f^{2} / 2$, which is independent of the $\phi_{a}$ fields.) With $S$ replaced by its vacuum value $S_{0}, \varnothing_{2}$ leads to the potential

$$
\mathcal{V}_{2}=-\mathcal{C} \frac{3 g^{2} \Lambda^{2}}{32 \pi^{2}} \operatorname{Tr}\left\{\left[t_{a}, S_{0}\right]\left[t_{a}, S_{0}\right]\right\}
$$

where $S_{0}$ is given by Eq. (27) and $\mathcal{C}$ is of unit magnitude. The contribution to $\mathcal{C}$ coming from the low energy theory cut off at $\Lambda$ is positive. We take this to be the case in general.

There are additional operators involving higher powers of $S$ but still bilinear in $t_{a}$. By making use of the expression (28), it can be seen that, in analogy to the $S O(3)$ model, they have the same dependence on the $\phi$ fields as $\varnothing_{2}$, up to an additive constant. They lead to potential terms that can be interpreted as simply redefining the parameters of $\mathcal{V}_{2}$, up to an additive constant. Thus $\mathcal{V}_{2}$ can be taken to describe the vacuum structure to order $g^{2}$. 


\section{Minimization of $\mathcal{V}_{2}$}

Since $F$ is diagonal, it can be shown that all but one of the 20 derivatives of $\mathcal{V}_{2}$ vanish at the origin $\hat{\phi}_{a}=0$. The exception is the derivative along $\hat{\phi}_{9}$ corresponding to the generator $s_{9}$, which has non-vanishing entries in only the ' 3,8 ' and ' 8,3 ' positions. To discuss the stable minima, it is convenient to adopt the ordering convention

$$
f_{8}<f_{4}<f_{5}<f_{1}<f_{2}<f_{6}<f_{7}<f_{3} .
$$

Then the potential has a stable minimum for vanishing values of all $\hat{\phi}_{a}$ for $a=11 \ldots 28$. With these choices, it is minimized in the $\hat{\phi}_{9}$ direction at a value depending on the elements of $F$. For the case $3 f_{1}+3 f_{2}+f_{3}+f_{8}>0$, it is at

$$
\left\langle\hat{\phi}_{9}\right\rangle=-\frac{1}{\sqrt{6}} \arctan \frac{\sqrt{3}\left(f_{4}+f_{5}-f_{6}-f_{7}\right)}{\left(3 f_{1}+3 f_{2}+f_{3}+f_{8}\right)} .
$$

For the case $3 f_{1}+3 f_{2}+f_{3}+f_{8}<0$, the minimum is at $\pi / \sqrt{6}$ plus this value.

At this minimum in all directions except $\hat{\phi}_{10}$, the potential is flat in $\hat{\phi}_{10}$. This is the direction associated with the generator $s_{10}$, defined such that the set $\left(t_{3}, t_{8}, s_{9}, s_{10}\right)$ forms a basis of the Cartan subalgebra of $S O(8)$.

The local minimum in all directions except $\hat{\phi}_{10}$ is not unique. Inspection shows that there are $96=2^{4} \times 3$ ! physically equivalent local minima. They are all flat in $\hat{\phi}_{10}$, and they correspond to values of the other $\hat{\phi}_{a}$ that simply reorder the elements of $F$ in $S_{0}$, exchanging $\left(f_{1}, f_{2}\right),\left(f_{4}, f_{5}\right),\left(f_{6}, f_{7}\right)$ and $\left(f_{3}, f_{8}\right)$ or permuting the pairs $\left(f_{1}, f_{2}\right),\left(f_{4}, f_{5}\right)$ and $\left(f_{6}, f_{7}\right)$. All of these physically equivalent minima are $C$-conserving; they correspond to $\hat{\phi}_{a}$ values such that $\left[C, S_{0}\right]=0$. This is evident with $\hat{\phi}_{a}=0(a=11, \ldots 28)$ and $\hat{\phi}_{9}$ given by Eq. (38), since $\hat{\phi}_{9}$ is a $C$ conserving direction $\left(\left[C, t_{9}\right]=0\right)$. With $f$ setting the scale for each of the 19 massive $\hat{\phi}$ 's, the mass of each of them is of order $g f$, the same as the gauge-boson mass. The flat direction $\hat{\phi}_{10}$ is, however, not $C$ conserving; thus the determination of the $C$ symmetry of the vacuum awaits further analysis of this direction.

The flatness in the $\hat{\phi}_{10}$ direction is not an accident. It can be understood in terms of the symmetries of the system. In any of the limits $f_{1} \rightarrow f_{2}, f_{4} \rightarrow f_{5}$, or $f_{6} \rightarrow$ $f_{7}$, an $S O(2)$ subgroup remains unbroken. Thus there will be one less PNGB in the spectrum. In each case, one can verify explicitly that at each local minimum the corresponding unbroken generator is a linear combination of $s_{10}, t_{3}$ and $t_{8}$. Since the latter two are the gauged generators, $\hat{\phi}_{3}$ and $\hat{\phi}_{8}$ will not develop a potential. Thus $\hat{\phi}_{10}$ is the PNGB that decouples in each case, and any potential term for $\hat{\phi}_{10}$, derived from polynomial functions of $S_{0}$, must be proportional to

$$
\left(f_{1}-f_{2}\right)\left(f_{4}-f_{5}\right)\left(f_{6}-f_{7}\right) .
$$

Since this expression is cubic in the $f^{\prime}$ 's, $\mathcal{V}_{2}$, which is bilinear in $S_{0}$ cannot exhibit $\hat{\phi}_{10}$ dependence.
Before proceeding, a cautionary remark is in order. We have noted that a large number of physically equivalent points exist, each a local minimum in all directions except the flat direction $\hat{\phi}_{10}$. A similar feature emerged in the analysis of the $S O(3)$ model. But unlike in that model, we have not yet explored the full domain of $\hat{\phi}$ values here 6 . We conjecture that, as in the $S O(3)$ case, there will be no further local minima degenerate with, or lower than, the ones we have identified.

\section{The $\hat{\phi}_{10}$ Direction}

To analyze the $\hat{\phi}_{10}$ direction, we turn to potential terms of order $g^{4}$. With $g$ small, they amount to small corrections to $\mathcal{V}_{1}$ for the massive degrees of freedom (all except $\left.\hat{\phi}_{10}\right)$. But they also induce a potential for $\hat{\phi}_{10}$. We study this $\hat{\phi}_{10}$ dependence by "integrating out" the other fields of the theory, all of which have mass scale $g f$, and focusing on energies $E \ll g f$. This amounts to setting each $\hat{\phi}_{a}$ in $S_{0}$, other than $\hat{\phi}_{10}$, equal to its VEV computed from $\mathcal{V}_{2}$.

We have noted that, in contrast to the $S O(3)$ model, there are no additional, $S U(3)$-invariant operators bilinear in $S$ that can lead to potential terms. But there are a variety of local operators generated at the $g^{4}$ level, and quartic in $S$, and they do give potentials with $\hat{\phi}_{10}$ dependence.

One of these, arising from the quantum loop expansion of the Lagrangian (25), is of special interest. It arises in Landau gauge from a pure-gauge-boson loop, second order in the gauge-boson mass operator (29). It enters with a coefficient proportional to $\ln \Lambda^{2}$, and gives a potential proportional to

$$
\operatorname{Tr}\left(\mathcal{M}^{2}(\hat{\phi})\right)^{2} \equiv \mathcal{M}^{2}(\hat{\phi})_{a b} \mathcal{M}^{2}(\hat{\phi})_{b a}
$$

where the gauge-boson mass matrix $\mathcal{M}^{2}(\hat{\phi})_{a b}$ is given by Eq. (30).

The one-loop computation leading to this potential also has logarithmic IR sensitivity, which is conveniently regulated at momenta of order $g f$ by summing all $\mathcal{M}^{2}(\hat{\phi})$ insertions to produce the one-loop Coleman-Weinberg potential. It takes the form

$$
\mathcal{V}_{C W}(\hat{\phi})=\frac{3}{64 \pi^{2}} \operatorname{Tr}\left[\left(\mathcal{M}^{2}(\hat{\phi})\right)^{2}\left(\ln \left(\frac{\mathcal{M}^{2}(\hat{\phi})}{\Lambda^{2}}\right)-\frac{1}{2}\right)\right],
$$

which incorporates the polynomial term (40). The $\ln \Lambda^{2}$ represents the contribution of unknown physics at energies above $\Lambda^{2}=16 \pi^{2} f^{2}$, also receiving contributions of the same order from all levels in the loop expansion of the effective low energy theory. We note that $\mathcal{V}_{C W}$ contains terms of order $g^{4} \ln g^{2}$.

The potential $\mathcal{V}_{C W}(\hat{\phi})$, after integrating out all fields except $\hat{\phi}_{10}$, provides nontrivial dependence on $\hat{\phi}_{10}$. We 
examine this behavior by first focusing on the piece polynomial in $\operatorname{Tr}\left(\mathcal{M}^{2}(\hat{\phi})\right)^{2}$. It leads to an effective potential as a function of $\hat{\phi}_{10}$ proportional to

$$
\frac{g^{4} \mathcal{D}}{16 \pi^{2}}\left(f_{1}-f_{2}\right)\left(f_{4}-f_{5}\right)\left(f_{6}-f_{7}\right)\left(f_{3}+f_{8}\right) \sin ^{2} \frac{3 \hat{\phi}_{10}}{\sqrt{2}},
$$

where $\mathcal{D}$ is a constant with unit magnitude. The extrema of this potential are at $\hat{\phi}_{10}=n \pi /(3 \sqrt{2})$ where $n$ is an integer. Each minimum corresponds to a mass of order $g^{2} f / 4 \pi \ll g f$, and each leads to an $S_{0}$ which, up to an $S U(3)$ transformation, commutes with $C$. Thus, whatever the sign of $\mathcal{D}$, this piece leads to a $C$-conserving vacuum.

The same is true of the term proportional to $\operatorname{Tr}\left(\mathcal{M}^{2}(\hat{\phi})\right)^{2} \ln \mathcal{M}^{2}(\hat{\phi})$. This can be seen by first noting that the gauge boson mass matrix $\mathcal{M}^{2}(\hat{\phi})$ has a $2 \times 2$ block-diagonal form when, as is the case here, only $\hat{\phi}_{9}$ and $\hat{\phi}_{10}$ are non-zero. Furthermore, only three of these blocks depend explicitly on $\hat{\phi}_{10}$. The eigenvalues of $\mathcal{M}^{2}(\hat{\phi})$ are then

$$
m_{ \pm}^{2 i}=a_{1}^{i} \pm \sqrt{a_{2}^{i 2}+a_{3}^{i} t}
$$

where $t=\sin ^{2} 3 \hat{\phi}_{10} / \sqrt{2}, i=1 \cdots 4$. The $a_{k}^{i}$ are functions of the $f_{a}$ parameters, with, in particular, $a_{3}^{4}=0$. Positivity and reality of the mass eigenvalues implies that $a_{2}^{i 2}+a_{3}^{i}>0$, and that $a_{1}^{i} \geq \sqrt{a_{2}^{i 2}+a_{3}^{i} t}$. One then finds (up to an additive constant):

$$
\mathcal{V}_{C W}(t)=\frac{3}{64 \pi^{2}} \sum_{i=1}^{3}\left(\left(m_{ \pm}^{2 i}\right)^{2}\left(\ln \frac{m_{ \pm}^{2 i}}{\Lambda^{2}}-\frac{1}{2}\right)\right) .
$$

Evaluating the second derivative of this expression with respect to $t$, we have

$$
\mathcal{V}_{C W}^{\prime \prime}(t)=\frac{3}{64 \pi^{2}} \sum_{i=1}^{3}\left(\frac{a_{3}^{i}}{y^{i} a_{1}^{i}}\right)^{2}\left(1+\frac{1}{2 y^{i}} \ln \frac{1-y^{i}}{1+y^{i}}\right),
$$

with $y^{i}=\sqrt{a_{2}^{i 2}+a_{3}^{i} t} / a_{1}^{i}$. In the allowed range $0 \leq$ $y^{i} \leq 1$, the $y^{i}$ dependent function is negative definite. Therefore $\mathcal{V}_{C W}^{\prime \prime}(t)$ is negative definite for any value $0 \leq$ $t \leq 1$, and hence the potential $\mathcal{V}_{C W}(t)$ has a minimum either at $t=0$ or $t=1$, where $C$ is unbroken.

In addition to this potential term, there are several others arising at order $g^{4}$ and $g^{4} \ln g^{2}$. We have checked that they, too, lead to a vacuum value $S_{0}$ that is $C$ conserving. These details will be described in a future paper.

\section{E. Higher Order Terms}

The key ingredient to induce spontaneous breaking of $C$ is the presence in the potential of terms proportional to $\sin ^{2 n} 3 \hat{\phi}_{10} / \sqrt{2}$, with more than one value of $n>0$.
This is analogous to the behavior found in the $S O(2)$ gauge theory. We have noted that up to $g^{4}$ order, only $n=1$ terms appear, and hence we must look to higher orders. Just as in the $S O(2)$ theory, special values of the symmetry-breaking parameters (here the $f_{a}$ ) can suppress the $\hat{\phi}_{10}$ dependence at order $g^{4}$, in which case terms higher order in $g$ can become comparable in strength and induce spontaneous breaking.

Operators leading to a potential with a sum of terms with different values of $n$ first appear at order $g^{6}$. They include at least six factors of the quantum field $S$. When such operators are used to generate potential terms through the replacement $S \rightarrow S_{0}$, and when all the fields $\hat{\phi}_{a}$ are integrated out except the very light $\hat{\phi}_{10}$, the potential includes terms such as $\sin ^{4} 3 \hat{\phi}_{10} / \sqrt{2}$ in addition to $\left(\sin ^{2} 3 \hat{\phi}_{10} / \sqrt{2}\right)$ behavior found at lower orders.

The interplay between these terms can lead to spontaneous breaking of $C$ just as the interplay of $V_{2}$ and $V_{4}$ did in the $S O(2)$ gauge theory. In analogy to that example, there are values of the $S O(8)$ symmetry breaking parameters (the $f_{a}$ 's) that naturally suppress the $\hat{\phi}_{10}$ dependence of the terms lower order in $g$, allowing the higher-order terms to compete and induce transitions to a vacuum state with broken $C$. Phase diagrams analogous to Fig. 1 can be constructed and used to describe the possible phases. The details of this study will be presented in a future paper. We conclude our discussion here with three comments reminiscent of those made in the case of the gauged $S O(2)$ model:

- The values of the $f_{a}$ 's that lead to the necessary suppression to allow $C$ breaking are hierarchical.

- The effective-theory framework is predictive with respect to $C$ breaking. That is, if the ( $C$-invariant) parameters of the effective Lagrangian below the scale $\Lambda$ are measured, then by making use of the phase diagram in these parameters, one can predict whether $C$ is spontaneously broken. We expect the intrinsic breaking to be of order unity as in the $S O(2)$ gauge theory.

- The spontaneous breaking of $C$ has physical consequences. As in the $S O(2)$ gauge theory, there is a simple selection rule if $C$ is unbroken: the $\phi_{10}$ particles are conserved mod 2. If $C$ is spontaneously broken, however, odd powers are allowed and there is no conservation of the $\phi_{10}$ particles. More interestingly in the present case, a $C$-violating value of $\hat{\phi}_{10}$ also enters the gauge boson mass matrix $\mathcal{M}^{2}(\hat{\phi})$. This has immediate consequences if the $S U(3)$ gauge theory is used as a flavor gauge theory coupled to the three families of quarks. With differing up- and down-type $S U(3)$ assignments, the parameter $\hat{\phi}_{10}$ becomes a measurable phase in the quark mass matrix. 


\section{CONCLUSIONS}

We have analyzed the spontaneous breaking of discrete symmetries in the effective low-energy description of a spontaneously broken gauge theory. We have focused on an $S U(3)$ gauge theory, as well as a simpler $S O(2)$ example. To describe the breaking, we embedded these gauge theories in models with larger global symmetry groups, $S O(8)$ and $S O(3)$ respectively. The motivation for this is that whatever the high energy physics producing the spontaneous breaking of the gauge group, it is likely to possess a larger global symmetry than the gauged one. Hence PNGB's are expected to arise, with masses controlled by the gauge coupling. Examples are (extended) technicolor and multi-Higgs models of flavor.

We constructed the effective theory below the breaking scale $\Lambda$ of the continuous symmetries, taking the gauge coupling small enough so that the particles of the theory are light compared to $\Lambda$. The particle content consists of the PNGB's produced by the spontaneous breaking of the approximate global continuous symmetries, and the (massive) gauge bosons. Small gauge coupling can be directly relevant to real-world gauged flavor symmetry providing that forces other than the gauge interaction are at least partly responsible for the breaking of the symmetry.

We studied whether the potential for the PNGB's, generated from the operators of the low-energy theory, can trigger the spontaneous breaking of the discrete symmetries. We found that in both models, potentials arising at leading orders in $g$ are minimized at discrete-symmetry preserving values of the PNGB VEV's. In the $S U(3)$ case, this happens at order $g^{2}$ for all PNGB fields except one $\left(\hat{\phi}_{10}\right)$. For the latter, it happens at order $g^{4}$ (and $g^{4} \ln g^{2}$ ). But operators generated at higher orders in the gauge coupling induce potentials that have a different functional dependence on the PNGB VEV's, one that can naturally lead to breaking.

For generic values of the parameters of the low energy theory, the dominance of the lower-order terms in $g$ does not allow the higher order terms to trigger discretesymmetry breaking. But this breaking becomes possi- ble if the parameters of the effective low energy theory are such as to suppress the PNGB-field dependence of the lower order terms. As we discussed in detail for the $S O(2)$ gauge theory, this requires a hierarchical set of scales in the effective theory.

There are lines of second order phase transitions in the parameter space, and the values of the symmetrybreaking order parameter in the broken phases is not typically small. Different phases, in which different symmetries are preserved, are characterized by observable properties, such as couplings that violate certain selection rules. The models are predictive, in the sense that the knowledge of the (discrete-symmetry preserving) parameters of the low energy theory allows one to determine in which phase the theory lies, and compute the magnitude of symmetry-breaking interactions.

The motivation for this study was the idea that measured CP-violation could naturally arise from the mechanism responsible for the generation of the flavor structure of the standard model, more specifically from the spontaneous breaking of a gauged flavor symmetry. Our conclusion is that the spontaneous breaking of discrete symmetries can arise purely from the gauge sector of such theories and that it is generically expected to be intrinsically large. This phenomenon can be analyzed within the framework of low-energy effective field theory, without knowledge of the details of the underlying theory and gauge-symmetry breaking, providing that the gauge coupling is sufficiently weak. The key necessary ingredient for discrete-symmetry breaking is the presence of hierarchies (small mixing angles) in the low-energy effective theory, allowing for otherwise suppressed operators to induce the spontaneous breaking.

\section{Acknowledgments}

We thank Kenneth Lane, Nicholas Read, Robert Shrock, and Witold Skiba for helpful conversations. This research was partially supported by a Department of Energy grant DE-FG02-92ER-40704.
[1] J. L. Chkareuli, JETP Lett. 32, 671 (1980) [Pisma Zh. Eksp. Teor. Fiz. 32, 684 (1980)]; Z. G. Berezhiani and J. L. Chkareuli, Sov. J. Nucl. Phys. 37, 618 (1983) [Yad. Fiz. 37, 1043 (1983)];

[2] Z. Berezhiani and A. Rossi, Nucl. Phys. B 594, 113 (2001) arXiv:hep-ph/0003084; A. Masiero, M. Piai, A. Romanino and L. Silvestrini, Phys. Rev. D 64, 075005 (2001) arXiv:hep-ph/0104101; G. G. Ross, L. VelascoSevilla and O. Vives, Nucl. Phys. B 692, 50 (2004) arXiv:hep-ph/0401064.

[3] A. E. Nelson, Phys. Lett. B 136, 387 (1984); S. M. Barr, Phys. Rev. Lett. 53, 329 (1984).

[4] T. Appelquist, M. Piai and R. Shrock, Phys. Rev.
D 69, 015002 (2004) arXiv:hep-ph/0308061; T. Appelquist, M. Piai and R. Shrock, Phys. Lett. B 593, 175 (2004) arXiv:hep-ph/0401114; T. Appelquist, M. Piai and R. Shrock, Phys. Lett. B 595, 442 (2004) arXiv:hep-ph/0406032; T. Appelquist, N. Christensen, M. Piai and R. Shrock, Phys. Rev. D 70, 093010 (2004) arXiv:hep-ph/0409035.

[5] A. Martin and K. Lane, Phys. Rev. D 71, 015011 (2005) arXiv:hep-ph/0404107; K. Lane and A. Martin, Phys. Rev. D 71, 076007 (2005) arXiv:hep-ph/0501204.

[6] L. J. Boya, Rep. Math. Phys. (Poland) 30, 149 (1991); L. J. Boya, arXiv:math-ph/0212067. 